\title{
Novel Method of Evaluating the Purity of Multiwall Carbon Nanotubes Using Raman Spectroscopy
}

\author{
Young Chul Choi, ${ }^{1}$ Kyoung-In Min, ${ }^{2}$ and Mun Seok Jeong ${ }^{3,4}$ \\ ${ }^{1}$ CNT Team, Hanwha Chemical, 80 Annamro 402 gil, Bupyeong-gu, Incheon 403-030, Republic of Korea \\ ${ }^{2}$ Spectro, Gwangju 500-712, Republic of Korea \\ ${ }^{3}$ Center for Integrated Nanostructure Physics, Institute for Basic Science, Sungkyunkwan University, \\ Suwon 440-746, Republic of Korea \\ ${ }^{4}$ Department of Energy Science, Sungkyunkwan University, Suwon 440-746, Republic of Korea
}

Correspondence should be addressed to Mun Seok Jeong; mjeong@skku.edu

Received 15 October 2013; Accepted 29 October 2013

Academic Editor: Clare C. Byeon

Copyright ( 2013 Young Chul Choi et al. This is an open access article distributed under the Creative Commons Attribution License, which permits unrestricted use, distribution, and reproduction in any medium, provided the original work is properly cited.

\begin{abstract}
We propose the quantitative method of evaluating the purity of multiwall carbon nanotubes (MWCNTs) using Raman spectroscopy. High purity MWCNTs were prepared by chemical vapor deposition (CVD) to be used as a reference material with $100 \%$ purity. Since the intensity and wavenumber of $D^{\prime \prime}$-band located at around $1500 \mathrm{~cm}^{-1}$ were found to be independent of the excitation wavelength of a laser, the purity of MWCNTs was measured by comparing the intensity ratio of $D^{\prime \prime}$-band to $G$-band $\left(I_{D^{\prime \prime}} / I_{G}\right)$ of the sample with that of a reference material. The established method was verified by testing the mixture of amorphous carbon particles and reference MWCNTs.
\end{abstract}

\section{Introduction}

Since multiwall carbon nanotubes (MWCNTs) possess high electrical conductivity, high thermal conductivity, and excellent mechanical properties [1-3], they have been extensively studied for being used in the electrical, thermal, and mechanical applications [4-7]. In order to achieve better characteristics of application products, the advancement in the synthesis technology has been tried to improve the properties of MWCNTs, including crystallinity, dispersibility, and purity [8-10]. Among these characteristics, purity is one of the most important properties from the viewpoint of better performance of application products. In addition, since higher purity means that a smaller amount of CNTs is required to achieve targeted properties, it is very important for industrial applications to use high purity MWCNTs in terms of low cost. Many researchers have used thermogravimetric analysis (TGA) [11-13] and $D$-band to G-band intensity ratio of Raman spectrum $\left(I_{D} / I_{G}\right)[14]$ to measure the purity of MWCNTs. However, TGA represents the whole carbon content in the sample because measuring the weight loss with increasing temperature does not distinguish carbon nanotubes from other carbon species. Meanwhile, apart from the fact that the intensity and position of $D$-band peak are varied with the excitation wavelength of a laser to a great extent [15], $D$-band originates from not only amorphous carbon particles but also nanotube-related aspects including defects in the curved graphene sheets, tube ends, and finite size of crystalline domains of the tubes [16]. Thus, the measurement of $\left(I_{D} / I_{G}\right)$ is not useful for evaluating the purity of MWCNTs using various wavelengths of laser. In contrast to $D$-band, the intensity and position of $D^{\prime \prime}$ band are not varied with the wavelength of laser. Moreover, $D^{\prime \prime}$-band originates from only amorphous carbons [17]. Therefore, we propose here the quantitative method evaluating the purity of MWCNTs using the $D^{\prime \prime}$-band Raman spectrum of MWCNTs as a signal for amorphous carbon particles.

As-synthesized MWCNTs are in general composed of the nanotubes, catalyst particles, and amorphous carbonaceous particles. Since the crystallinity of MWCNTs observed from Raman spectroscopy is clearly distinguishable from that of amorphous carbons, we used the comparison of Raman peaks 


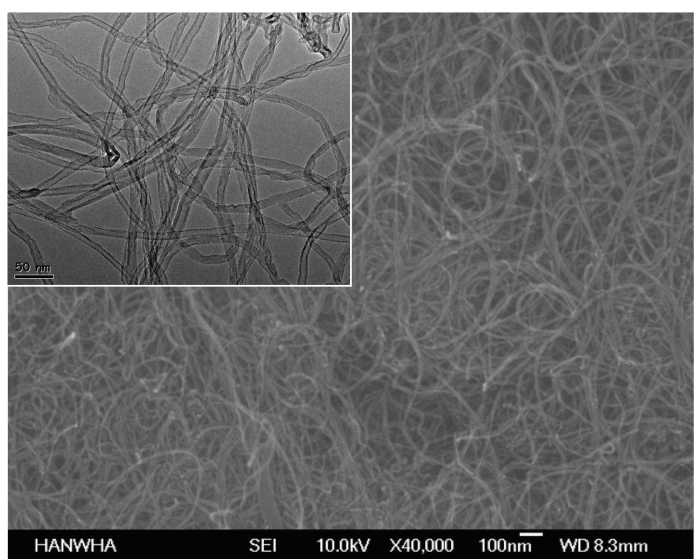

(a)

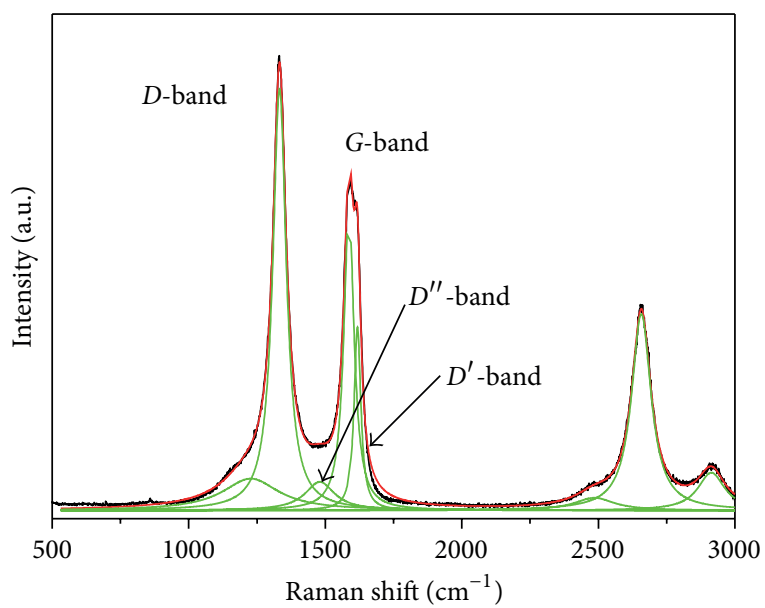

(b)

FIGURE 1: (a) SEM image of reference MWCNTs with 100\% purity. Inset shows the HRTEM image of reference MWCNTs. (b) Raman spectrum of reference MWCNTs.

observed from MWCNTs and amorphous carbons for evaluating the purity of MWCNTs. A high purity MWCNT-sample was prepared and then used as a reference material. The purity was evaluated from comparing $D^{\prime \prime}$-band to $G$-band intensity ratio $\left(I_{D^{\prime \prime}} / I_{G}\right)$ of sample with $I_{D^{\prime \prime}} / I_{G}$ of reference materials. MWCNTs have been synthesized by either arcdischarge $[18,19]$ or chemical vapor deposition (CVD) [110]. Since the MWCNTs synthesized by arc-discharge have higher crystallinity, $D^{\prime \prime}$-band is scarcely seen in the Raman spectrum. Therefore, the method to be introduced in this report is useful for only CVD-grown MWCNTs. But, the MWCNTs synthesized by arc-discharge are not used in the practical applications due to the impossibility of mass production. Therefore, it is still worthy of developing the method have evaluating the purity of CVD-grown MWCNTs that are unexceptionally shown $D^{\prime \prime}$-band in the Raman spectrum. We will provide the detailed protocols for determining the purity of CVD-grown MWCNTs, which are confirmed by testing the mixture of high purity MWCNTs and amorphous carbon particles.

\section{Experimental}

In order to synthesize MWCNTs, $\mathrm{Fe} / \mathrm{Mo} / \mathrm{Al}_{2} \mathrm{O}_{3}$ particles were prepared using combustion method. Fe/Mo particles were used as catalysts and $\mathrm{Al}_{2} \mathrm{O}_{3}$ was supporting the catalysts. Metal-organic precursors of iron nitrate, molybdenum acetate, and aluminum nitrate were dissolved in deionized water, and then the prepared solution was transformed into catalyst powder by thermal treatment at $450^{\circ} \mathrm{C}$ for $30 \mathrm{~min}$ in air ambient. The quartz boat having the catalyst powder was placed inside a quartz tube reactor. After heating the reactor to $700^{\circ} \mathrm{C}$ in Ar atmosphere, a gas mixture of $\mathrm{C}_{2} \mathrm{H}_{4} / \mathrm{H}_{2}$ $(1: 1)$ was fed into the reactor with a pressure of 1 atm for the synthesis, followed by cooling to room temperature under Ar atmosphere.

The morphologies of MWCNTs were investigated using scanning electron microscopy (SEM, JEOL JSM 6700F) and transmission electron microscopy (TEM, FEI Tecnai 20, $200 \mathrm{kV}$ ). The amount of catalyst particles included in the synthesized MWCNT samples was investigated by TGA. Amorphous carbon powder was purchased from Aldrich to be used as an impurity material. In order to verify the proposed evaluation method, several intermediate purity samples, having the purity range of $0-100 \%$ with $20 \%$ interval, were prepared by mixing high purity MWCNTs and amorphous carbon powder, considered as $100 \%$ and $0 \%$, respectively. Then, Raman spectroscopy measurement was carried out to evaluate the purity of MWCNTs using a Raman microscope (TRIVIA, Acton).

\section{Results and Discussion}

Through optimizing the experimental parameters for the synthesis of MWCNTs, we prepared very high purity MWCNTs, as can be seen in Figure 1(a). Despite of a number of observations using SEM, we could not see carbonaceous particles. TEM image (inset figure) clearly shows the synthesized materials are all of MWCNTs with hollow insides. According to TGA analysis, two weight-percent (wt\%) of catalyst metal particles is included in the prepared MWCNTs. However, we could hardly see the catalyst particles during both SEM and TEM observations. It is believed that $2 \mathrm{wt} \%$ of catalyst particles corresponds to an extremely small volume percent that could be negligible, because the bulk density of catalyst particle is much larger than that of MWCNTs. Furthermore, most of the catalyst particles are residing inside MWCNTs after the synthesis [20], which does not affect Raman spectrum. Therefore, this high purity MWCNTs could be considered as a reference material with $100 \%$ purity. Figure 1(b) represents the Raman spectrum of a reference MWCNT sample. The spectrum was taken using an excitation wavelength of $633 \mathrm{~nm}$. The Raman spectrum shows a typical feature of MWCNTs: the first ordered $D^{-}, G^{-}$, and $D^{\prime}$-band peaks located at around 1350, 1582, and $1610 \mathrm{~cm}^{-1}$, respectively. $G$-band peak is assigned to "in plane" displacement of the carbons 


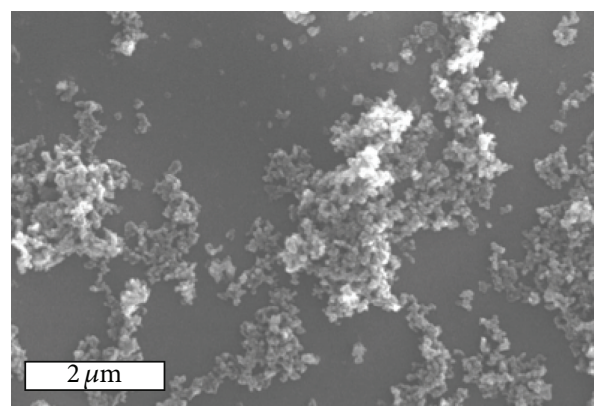

(a)

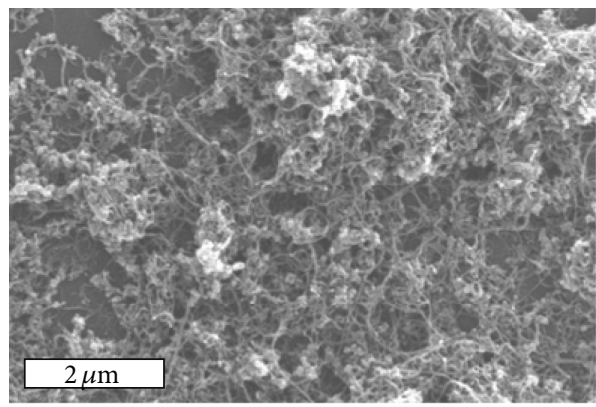

(c)

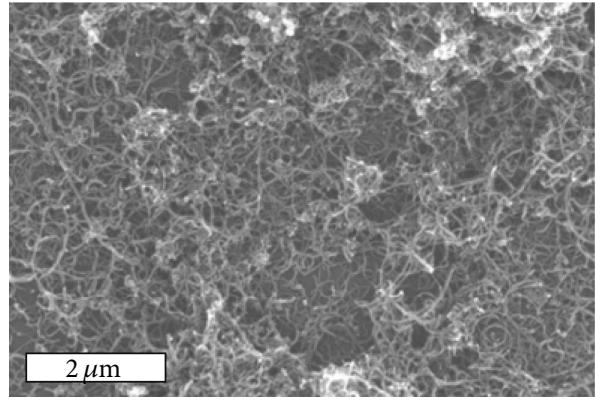

(e)

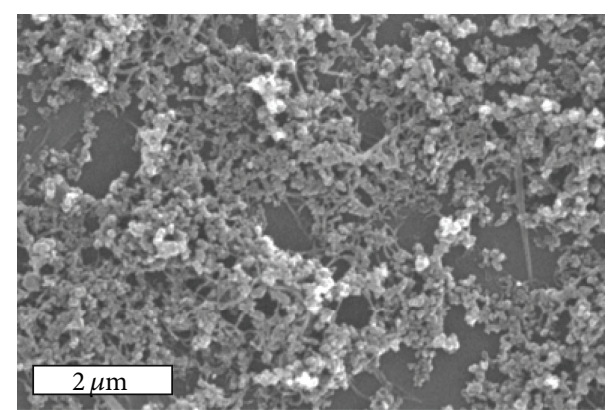

(b)

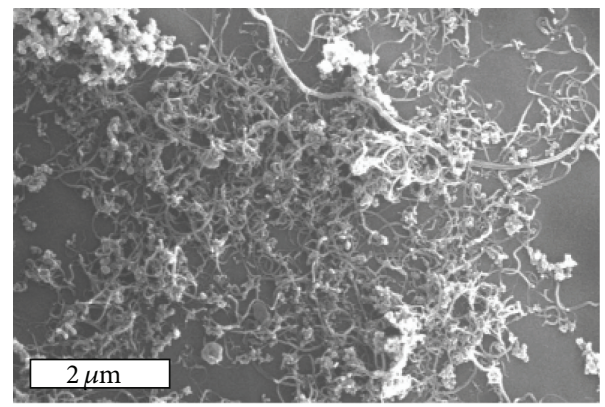

(d)

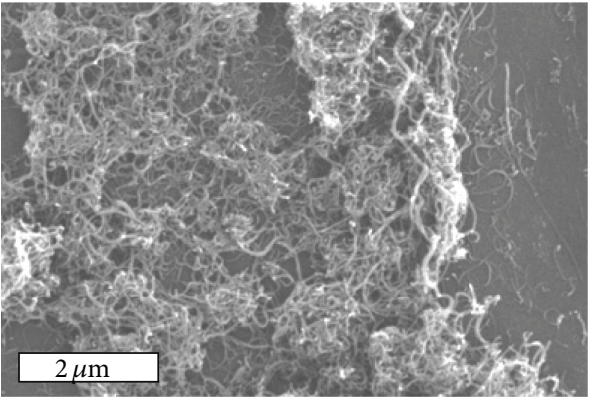

(f)

FIGURE 2: SEM images of purity-controlled MWCNT samples by mixing amorphous carbon particles and highly pure MWCNTs: (a) 0\%, (b) $20 \%$, (c) $40 \%$, (d) $60 \%$, (e) $80 \%$, and (f) $100 \%$.

strongly coupled in the hexagonal sheets [17]. D- and $D^{\prime}$ band are found when disorder is introduced into the graphite structure. For this reason, the intensity ratio of $D$-band to $G$ band $\left(I_{D} / I_{G}\right)$ has been used to determine the crystallinity of MWCNTs [15, 20]. However, according to Behler et al. [15], the intensity and position of $D$-band peak are varied with the excitation wavelength of laser to a great extent. Therefore, the measurement of $I_{D} / I_{G}$ cannot be used as a standard method for evaluating the crystallinity of MWCNTs although $I_{D} / I_{G}$ is used to compare the crystallinity of carbon nanotubes when the identical wavelength is used. Another broad peak ( $D^{\prime \prime}$-band) located at around $1500 \mathrm{~cm}^{-1}$ is also found in the Raman spectrum (Figure 1(b)). $D^{\prime \prime}$-band was reported to be associated with amorphous $s p^{2}$-bonded forms of carbon $[21,22]$. Unlike $I_{D} / I_{G}$, the intensity ratio of $D^{\prime \prime}$-band to $G^{-}$ band $\left(I_{D^{\prime \prime}} / I_{G}\right)$ was found to be invariable with the excitation wavelength of laser. In addition, $D^{\prime \prime}$-band is associated with only amorphous carbons. Hence, we suggest $I_{D^{\prime \prime}} / I_{G}$ instead of $I_{D} / I_{G}$ to be used for representing the crystallinity of carbon nanotubes. $I_{D^{\prime \prime}} / I_{G}$ of the reference samples obtained from Figure $1(\mathrm{~b})$ is 0.06 . On the other hand, $I_{D^{\prime \prime}} / I_{G}$ of amorphous carbon particles that was purchased from Aldrich is 0.37 . We suggest that $I_{D^{\prime \prime}} / I_{G}$ of the high purity MWCNTs (0.06) and amorphous carbons (0.37) should represent the purities of $100 \%$ and $0 \%$, respectively. Therefore, the purity of $\operatorname{MWCNTs}\left(P_{\mathrm{MW}}\right)$ can be simply determined by an equation of

$$
P_{\mathrm{MW}}(\%)=\left(\frac{\mathrm{AC}\left(D^{\prime \prime} / G\right)-\mathrm{MW}\left(D^{\prime \prime} / G\right)}{\operatorname{AC}\left(D^{\prime \prime} / G\right)-\operatorname{RMW}\left(D^{\prime \prime} / G\right)}\right) \times 100,
$$

where $\operatorname{AC}\left(D^{\prime \prime} / G\right), \operatorname{MW}\left(D^{\prime \prime} / G\right)$, and $\operatorname{RMW}\left(D^{\prime \prime} / G\right)$ are $D^{\prime \prime} / G$ ratios of amorphous carbon particles, MWCNTs being tested, and reference MWCNTs, respectively. In order to verify the suggested equation, intermediate purity samples were prepared with $20 \%$ purity interval from $0 \%$ to $100 \%$ by mixing high purity MWCNTs and amorphous carbon particles. 


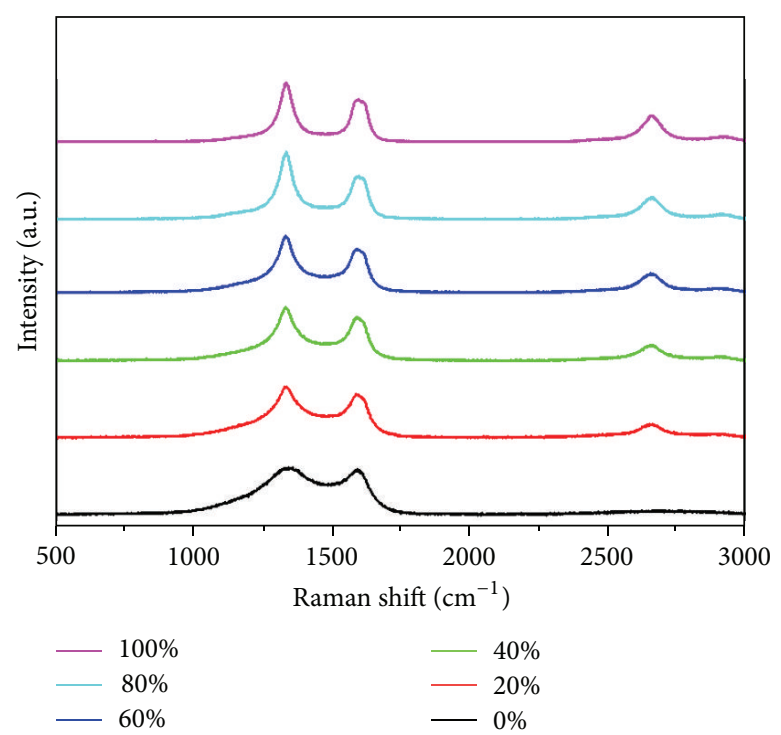

(a)

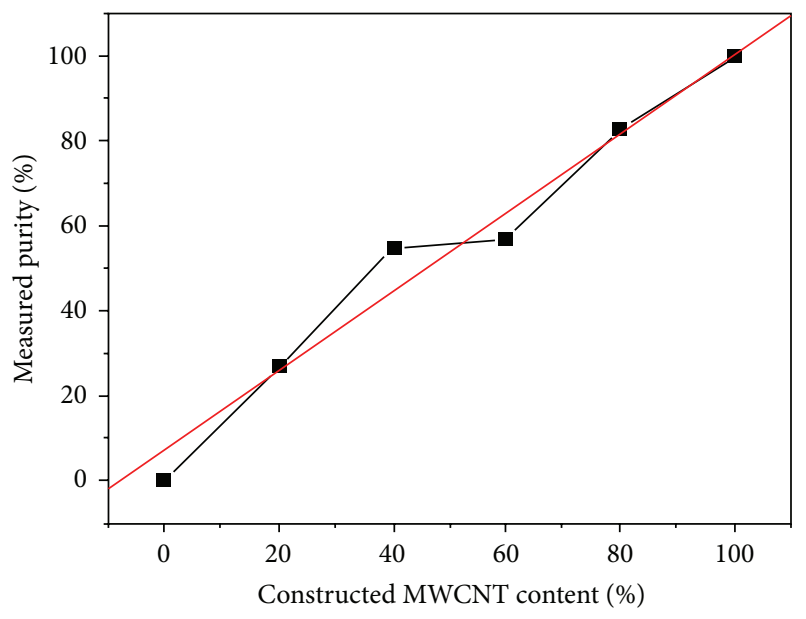

(b)

FIGURE 3: (a) Raman spectra, taken using an excitation wavelength of $633 \mathrm{~nm}$, of purity-controlled MWCNT samples with purities ranging from $0 \%$ to $100 \%$ with $20 \%$ interval. (b) Purity measured from Raman spectroscopy using 633 nm laser versus constructed content of MWCNTs.

First, we dispersed the MWCNTs and amorphous carbon particles in the dimethylformamide (DMF) separately with $1 \mathrm{mg} / 100 \mathrm{ml}$ concentration. Then, two solutions were mixed with designed ratios. The mixed solutions were dropped onto glass and Si substrates for the analyses by Raman and SEM, respectively. Figure 2 shows the SEM images of several intermediate purity samples. It can be clearly observed from these figures that the amount of impurity decreases as the purity of MWCNT increases. However, it is hard to define the purity of samples quantitatively by using SEM measurements.

Six different intermediate samples prepared with different purities $(0 \%-100 \%)$ were analyzed by Raman spectroscopy. Figure 3(a) shows the Raman spectra, taken using an excitation wavelength of $633 \mathrm{~nm}$, of MWCNTs with various purities. $100 \%$ and $0 \%$ purity samples show the Raman spectra of reference MWCNTs and amorphous carbons, respectively. Although the deconvoluted peaks of $0 \%$ purity sample are not presented here, it was found that the Raman spectrum of $0 \%$ purity sample is composed of not only conventional $D$ and $G$-bands but also $D^{\prime \prime}$-band with a considerable intensity. With increasing the purity of MWCNTs, full width at the half maximum (FWHM) of the peak at $1200-1400 \mathrm{~cm}^{-1}(D+$ $D^{\prime \prime}$-bands) decreases because the contribution of $D^{\prime \prime}$-band is getting smaller. We applied the proposed equation to the series of MWCNT samples with intentionally varied purity, and the results are illustrated in Figure 3(b). Figure 3(b) plots the purity evaluated using the equation to the constructed contents with varying purities from $0 \%$ to $100 \%$. The linear fitting yielded a slope close to unity, 0.96 , and the confidence value $R^{2}$ was 0.95 . Thus, the evaluated purities of MWCNTs are in good agreement with the designed values of constructed content. It can be therefore said that the suggested equation can be simply used for evaluating the purity of MWCNTs.

Various lasers with different excitation wavelengths are used in Raman spectroscopy. Therefore, in order to standardize an evaluation method using Raman spectroscopy, the method should not be dependent upon the excitation wavelength. A number of previous literatures reported the crystallinity of carbon nanotubes determined from the intensity ratio of $D$-band $\left(\sim 1350 \mathrm{~cm}^{-1}\right)$ to $G$-band $\left(I_{D} / I_{G}\right)$. However, the measurement of $I_{D} / I_{G}$ cannot be used as a standardized evaluation method since the intensity of $D$ band is significantly dependent upon the wavelength of laser [15]. In this study, we measured the purities of several different samples by using the excitation wavelength of not only $633 \mathrm{~nm}$ shown in Figure 3(b) but also $532 \mathrm{~nm}$ to verify the independency of this method on the wavelength. Generally, $633 \mathrm{~nm}$ and $532 \mathrm{~nm}$ laser are widely used for the Raman scattering measurement of MWCNTs. Figure 4 represents the purities evaluated from the Raman spectra taken using $532 \mathrm{~nm}$ laser as a function of constructed content. $I_{D^{\prime \prime}} / I_{G}$, obtained using an excitation wavelength of $532 \mathrm{~nm}$, of high purity reference MWCNTs and amorphous carbon particles was 0.07 and 0.49 , respectively. Although $I_{D^{\prime \prime}} / I_{G}$ measured using $532 \mathrm{~nm}$ laser is slightly different from that obtained using $633 \mathrm{~nm}$ laser, the purity measured using an excitation wavelength of $532 \mathrm{~nm}$ is also well matched to the constructed content, as can be seen in Figure 4. The linear fitting yielded a slope of 1.03 , and the confidence value $R^{2}$ was 0.96 . Thus, the purities of MWCNTs evaluated using excitation wavelength of $532 \mathrm{~nm}$ are also in good agreement with the designed values of constructed content. It can be therefore concluded that evaluating the purity of MWCNTs using $I_{D^{\prime \prime}} / I_{G}$ is valid regardless of excitation wavelength of laser. 


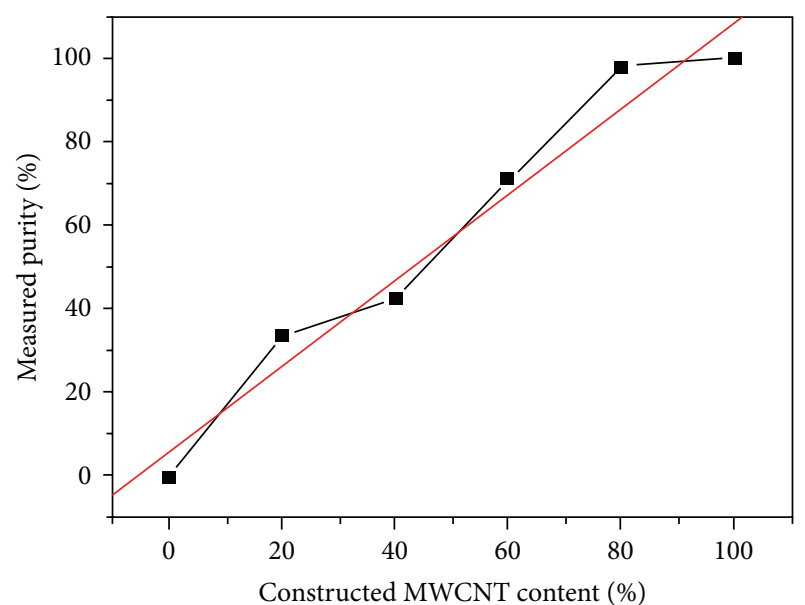

FIgURE 4: Purity measured from Raman spectroscopy using an excitation wavelength of $532 \mathrm{~nm}$ versus constructed content of MWCNTs.

\section{Conclusion}

A quantitative method for evaluating the purity of MWCNTs using Raman spectroscopy was newly proposed. The purity of MWCNTs was determined by comparing the intensity ratio of $D^{\prime \prime}$-band to $G$-band $\left(I_{D^{\prime \prime}} / I_{G}\right)$ of MWCNTs being tested with that of the high purity reference material. The established method was verified by testing the mixture of reference MWCNTs of high purity and amorphous carbon particles. Furthermore, it was found that the proposed method was found to be valid regardless of the excitation wavelength of laser.

\section{Acknowledgment}

This work was supported by Institute for Basic Science (IBS) (EM1304).

\section{References}

[1] L. J. Cui, H. Z. Geng, W. Y. Wang, L. T. Chen, and J. Gao, "Functionalization of multi-wall carbon nanotubes to reduce the coefficient of the friction and improve the wear resistance of multi-wall carbon nanotube/epoxy composites," Carbon, vol. 54, pp. 277-282, 2013.

[2] Y. J. Noh, H. S. Kim, and S. Y. Kim, "Improved electrical conductivity of a carbon nanotube mat composite prepared by in-situ polymerization and compression molding with compression pressure," Carbon Letters, vol. 13, no. 4, pp. 243-247, 2012.

[3] Y. C. Choi, Y. M. Shin, Y. H. Lee et al., "Controlling the diameter, growth rate, and density of vertically aligned carbon nanotubes synthesized by microwave plasma-enhanced chemical vapor deposition," Applied Physics Letters, vol. 76, no. 17, pp. 23672369, 2000.

[4] S. Akita, Y. Ohshima, and T. Arie, "Nanoincandescent consisting of individual carbon nanotubes," Applied Physics Express, vol. 4, no. 2, Article ID 025101, 2011.

[5] C. T. Chang, C.-P. Juan, and H.-C. Cheng, "Pillar height dependence of field-emission properties in an array of carbon nanotube pillars," Japanese Journal of Applied Physics, vol. 52, no. 18, Article ID 085101, 2013.

[6] A. Mierczynska, M. Mayne-L'Hermite, G. Boiteux, and J. K. Jeszka, "Electrical and mechanical properties of carbon nanotube/ultrahigh-molecular-weight polyethylene composites prepared by a filler prelocalization method," Journal of Applied Polymer Science, vol. 105, no. 1, pp. 158-168, 2007.

[7] F. L. Jin and S. J. Park, "Recent advances in carbon-nanotubebased epoxy composites," Carbon Letters, vol. 14, no. 1, pp. 1-13, 2013.

[8] J. S. Kim, S. J. Cho, K. S. Jeong, Y. C. Choi, and M. S. Jeong, "Improved electrical conductivity of very long multiwalled carbon nanotube bundle/poly (methyl methacrylate) composites," Carbon, vol. 49, no. 6, pp. 2127-2133, 2011.

[9] Y. C. Choi, Y. M. Shin, S. C. Lim et al., "Effect of surface morphology of $\mathrm{Ni}$ thin film on the growth of aligned carbon nanotubes by microwave plasma-enhanced chemical vapor deposition," Journal of Applied Physics, vol. 88, no. 8, pp. 48984903, 2000.

[10] Y. S. Park, H. S. Moon, M. Huh et al., "Synthesis of aligned and length-controlled carbon nanotubes by chemical vapor deposition," Carbon Letters, vol. 14, no. 2, pp. 99-104, 2013.

[11] P. Mahanandia, P. N. Vishwakarma, K. K. Nanda et al., "Synthesis of multi-wall carbon nanotubes by simple pyrolysis," Solid State Communications, vol. 145, no. 3, pp. 143-148, 2008.

[12] J. H. Lehman, M. Terrones, E. Mansfield, K. E. Hurst, and V. Meunier, "Evaluating the characteristics of multiwall carbon nanotubes," Carbon, vol. 49, no. 8, pp. 2581-2602, 2011.

[13] J. P. C. Trigueiro, G. G. Silva, R. L. Lavall et al., "Purity evaluation of carbon nanotube materials by thermogravimetric, TEM, and SEM methods," Journal of Nanoscience and Nanotechnology, vol. 7, no. 10, pp. 3477-3486, 2007.

[14] R. A. DiLeo, B. J. Landi, and R. P. Raffaelle, "Purity assessment of multiwalled carbon nanotubes by Raman spectroscopy," Journal of Applied Physics, vol. 101, no. 6, Article ID 064307, 2007.

[15] K. Behler, S. Osswald, H. Ye, S. Dimovski, and Y. Gogotsi, "Effect of thermal treatment on the structure of multi-walled carbon nanotubes," Journal of Nanoparticle Research, vol. 8, no. 5, pp. 615-625, 2006.

[16] W. Li, H. Zhang, C. Wang et al., "Raman characterization of aligned carbon nanotubes produced by thermal decomposition of hydrocarbon vapor," Applied Physics Letters, vol. 70, no. 20, pp. 2684-2686, 1997.

[17] T. Jawhari, A. Roid, and J. Casado, "Raman spectroscopic characterization of some commercially available carbon black materials," Carbon, vol. 33, no. 11, pp. 1561-1565, 1995.

[18] Y. S. Park, Y. C. Choi, K. S. Kim et al., "High yield purification of multiwalled carbon nanotubes by selective oxidation during thermal annealing," Carbon, vol. 39, no. 5, pp. 655-661, 2001.

[19] X. Song, Y. Liu, and J. Zhu, "Multi-walled carbon nanotubes produced by hydrogen DC arc discharge at elevated environment temperature," Materials Letters, vol. 61, no. 2, pp. 389-391, 2007.

[20] Z. Balogh, G. Halasi, B. Korbély, and K. Hernadi, "CVDsynthesis of multiwall carbon nanotubes over potassium-doped supported catalysts," Applied Catalysis A, vol. 344, no. 1-2, pp. 191-197, 2008. 
[21] D. S. Knight and W. B. White, "Characterization of diamond films by Raman spectroscopy," Journal of Materials Research, vol. 4, no. 2, pp. 385-393, 1989.

[22] P. V. Huong, "Structural studies of diamond films and ultrahard materials by Raman and micro-Raman spectroscopies," Diamond and Related Materials, vol. 1, no. 1, pp. 33-41, 1991. 

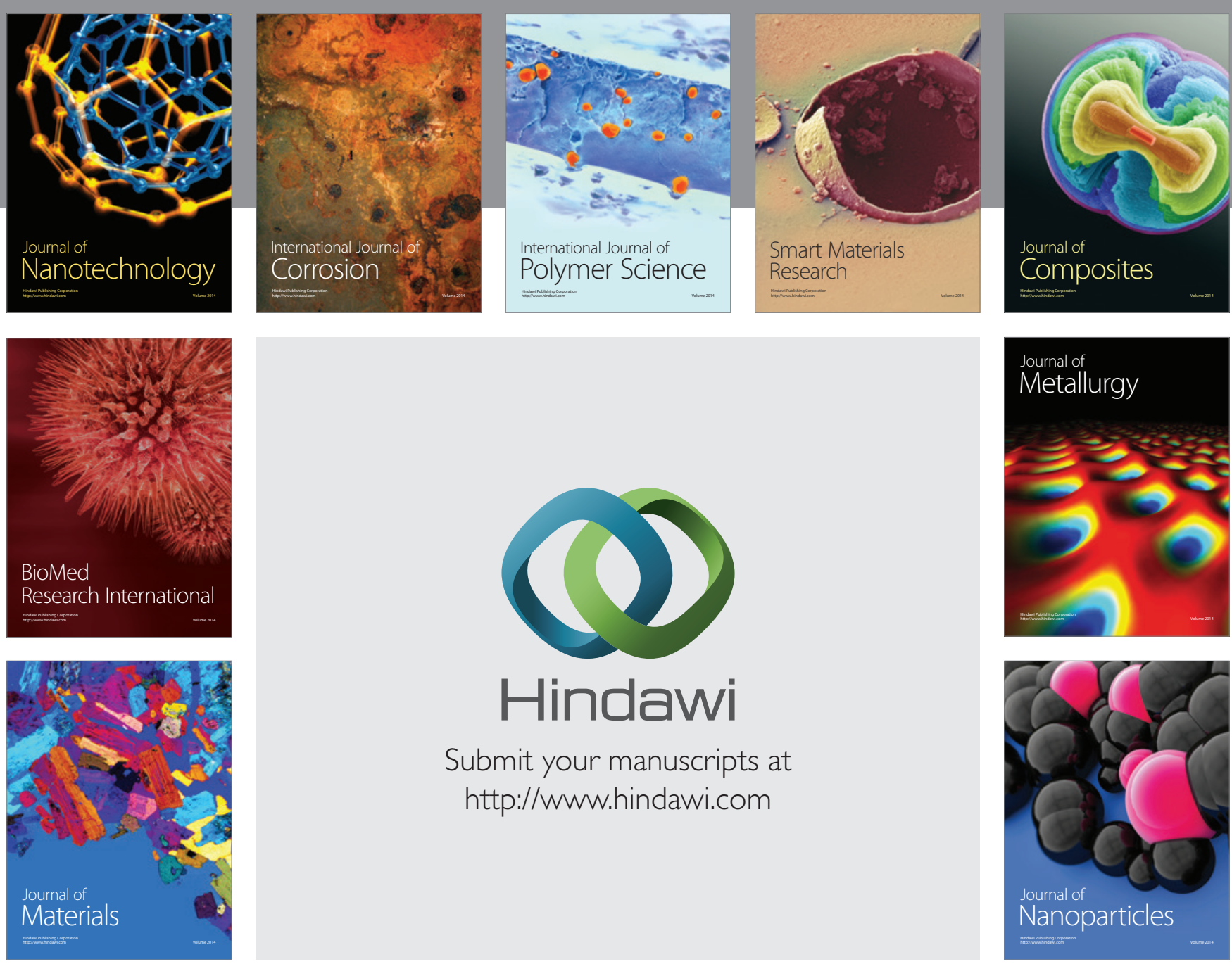

Submit your manuscripts at http://www.hindawi.com
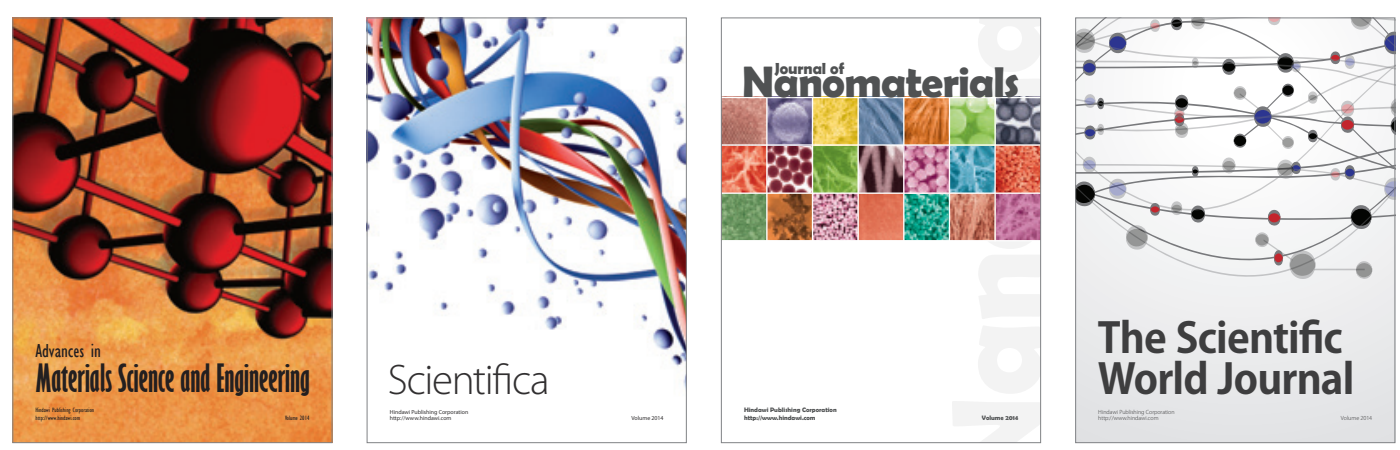

\section{The Scientific World Journal}
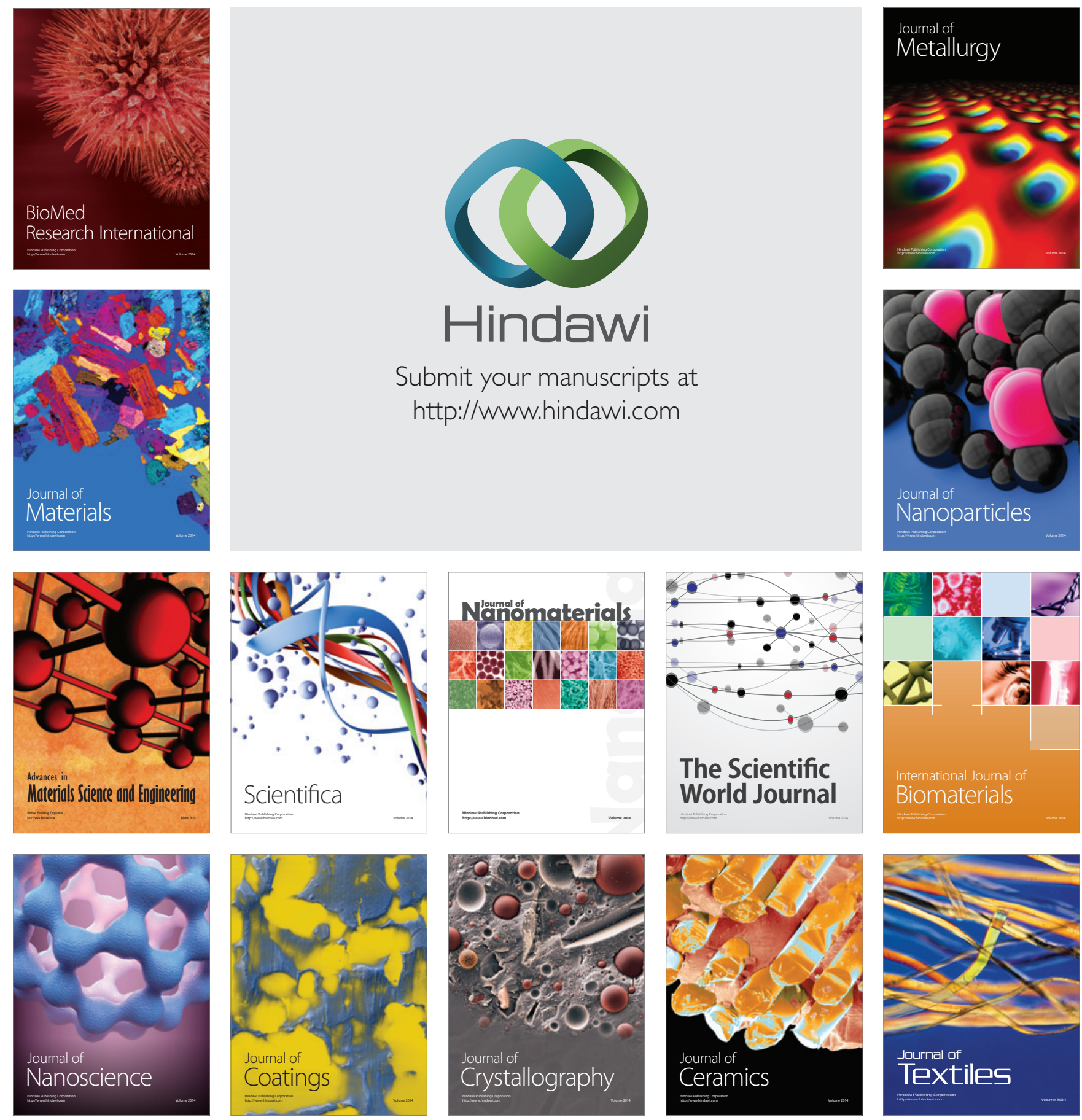